\title{
SÓCRATES: GESTO Y PALABRA POLÍTICA
}

\author{
Gustavo Gómez Pérez \\ doi:10.11144/Javeriana.uph34-69.sgpp
}

\section{RESUMEN}

La tesis central del artículo es que para Sócrates la virtud política se realiza exclusivamente en la esfera del lenguaje o el gesto. La argumentación consta de tres partes. En primer lugar, siguiendo las lecciones tituladas ¿Quésignifica pensar?, examino la interpretación heideggeriana de la figura de Sócrates en relación con los temas del gesto y lenguaje. Al concluir este análisis planteo que Heidegger omite aspectos importantes de la comprensión socrática de la relación entre virtud política y habla. Seguidamente, con base en el análisis de un pasaje central de la Apología de Platón, argumento que la virtud exige la comunicabilidad pública de la palabra, y se impone en el lenguaje y los gestos como un principio de acción que precede y excede al saber conceptual. Finalmente, concluyo que el poder político se restringe a la medialidad del lenguaje, a lo que la palabra hace o dice por sí misma.

Palabras clave: Sócrates; Martin Heidegger; gesto; lenguaje; virtud; política

Pontificia Universidad Javeriana, Bogotá, Colombia.

Correo electrónico: gustavo-gomez@javeriana.edu.co

Para citar este artículo: Gómez Pérez, G. (2017). Sócrates: gesto y palabra política. Universitas Philosophica, 34(69), pp. 173-194. ISSN 0120-5323, ISSN en línea 2346-2426. doi:10.11144/ Javeriana.uph34-69.sgpp

Una primera versión de este texto fue presentada como lección inaugural de la Facultad de Filosofía de la Pontificia Universidad Javeriana el 4 de agosto de 2016. 


\title{
SOCRATES: GESTURE AND POLITICAL SPEECH
}

\author{
Gustavo Gómez Pérez
}

\begin{abstract}
The main thesis of this paper is that for Socrates political virtue is accomplished exclusively in the sphere of pure language or gesture. The argument consists of three parts. First, following on from the lectures entitled What is Called Thinking?, I examine Heidegger's interpretation of the figure of Socrates in relation to the themes of language and gesture. Following on from this analysis, I remark that Heidegger overlooks important aspects of Socrates' understanding of the relation between political virtue and speech. Subsequently, based on the analysis of a central passage from Plato's Apology, I argue that virtue requires the public communicability of speech, and that it imposes itself over language and gestures as a principle of action that precedes and exceeds conceptual knowledge. Finally, I conclude by stating that political power is restricted to the medium of language, to what language itself does or says.
\end{abstract}

Key words: Socrates; Martin Heidegger; gesture; language; politics; virtue 


\section{La lectura heideggeriana sobre el gesto y el silencio de Sócrates}

EN SUS LECCIONES DE 1951 sobre ¿Qué significa pensar?, Heidegger (2002) afirma que Sócrates es el "pensador más puro de Occidente" (der reinste Denker des Abendlandes) y sugiere que aquello que lo caracteriza de manera superlativa es el hecho de situarse (sich [...] stellen) y mantenerse en la corriente (in den $\mathrm{Zu}$ gwind dieses Zuges) de lo que se sustrae (das Sichentziehende), esto es, de aquello que propiamente da que pensar (was eigentlich zu denken gibt), lo que llama al pensamiento (2002, pp. 19-20; 2005a, pp. 79-80) ${ }^{1}$.

Si bien la afirmación heideggeriana es sugerente, no es claro lo que implica en este caso la referencia a la pureza de un pensador como Sócrates, ni el uso del superlativo, ni, mucho menos, cómo debemos concebir aquello que llama propiamente al pensamiento y que se define como una corriente que se sustrae. Por este motivo, comenzaremos por explicitar lo que este pasaje sugiere sobre la pureza de Sócrates como pensador para, a partir de allí, en la segunda y tercera partes del presente artículo, proporcionar una interpretación sobre el carácter político del pensamiento socrático.

En primer lugar, al interpretar el pasaje introductorio, cabe subrayar que, literalmente, Heidegger alude a una corriente de aire. El término que utiliza es Zugwind y, teniendo en cuenta que en el curso de estas mismas lecciones se afirma que el medium para el pensamiento es el lenguaje, tal parece que la corriente en cuestión es un aliento fugaz como el de la voz, el habla ${ }^{2}$. La afirmación de Heidegger insinúa, además, que la particularidad de Sócrates como pensador se deriva de su capacidad para mantenerse firmemente anclado a algo inestable -con todo lo que implica esta paradoja-: él piensa aquello que, como el habla, es en cierto sentido invisible e inaprehensible. En este caso, entonces, la corriente del pensamiento se identifica con el ejercicio del habla y, más en general, con el lenguaje vivo, encarnado del gesto.

1 Las referencias a las obras de Heidegger se harán primero citando la versión en alemán de la Gesamtausgabe y, seguidamente, la traducción al español.

2 En el curso del presente artículo se usarán indistintamente los términos "lenguaje”, "habla" y "palabra”. 
Estas referencias a Sócrates surgen en una lección en cuyo núcleo argumentativo se afirma que el hombre solamente piensa en tanto que habla (nur insofern der Mensch spricht, denkt er) y en el que se conciben los gestos humanos como permeados integralmente por el lenguaje, aunque se trate de un lenguaje que se cualifica como silencioso. Específicamente, Heidegger (2002, p. 79; 2005a, p. 17) ${ }^{3}$ afirma lo siguiente: "[L]os gestos de la mano pasan por doquier a través del lenguaje, y pasan a su través en la forma más pura cuando el hombre habla precisamente callando" (Aber die Gebärden der Hand gehen überall durch die Sprache hindurch und zwar gerade dann am reinsten, wenn der Mensch spricht, indem er schweigt).

Este pasaje anticipa la valoración de Sócrates como el pensador más puro y correlaciona la pureza con el silencio, entendido como una modalidad gestual del decir, lo que se podría cualificar como un "puro" acto de expresión cuya significación permanece abierta, latente. En este punto de la lección, Heidegger hace referencia a las manos que se juntan, presumiblemente, en un gesto de piedad y oración ${ }^{4}$, una acción que sin decir nada específico evoca una actitud o disposición existencial que es también significativa: el tener las manos juntas mientras se reza no agrega nada al significado proposicional de la oración, pero testimonia una actitud de recogimiento que armoniza con el decir de la misma. La pureza refiere, pues, al silencio que es lenguaje y que está imbricado en un comportamiento, en un modo particular de ser y de estar en el mundo.

El término alemán que usa Heidegger para referir a los gestos es Gebärden y no Geste, el cual, en su forma verbal refiere al comportamiento en general, lo que nos permite suponer que en este caso se entiende todo comportamiento como gestualidad y expresión, esto es, como despliegue incipiente del lenguaje. Así pues, si el comportamiento en cuanto tal es gesto e indicación, es ya un comienzo del lenguaje, entonces su expresividad no está subordinada a una significación ulterior, trascendente, respecto de su carácter sensible. Por ello, todo gesto, como todo decir, es capaz de decir algo desde su propia medialidad o materialidad. De

3 La cursiva es mía, traducción modificada.

4 Esto es, precisamente, lo que recalca Jacques Derrida (1987) en su análisis del tema de la "mano" en Heidegger, criticando el énfasis en lo propio y la auto-afección que predomina en esta interpretación (p. 175). 
acuerdo con esto, para Heidegger lo que hace que Sócrates sea un pensador puro o, más precisamente, lo que determina la pureza de su pensamiento, es su relación con el lenguaje, su capacidad para mantenerse en la corriente viva de lo que el lenguaje por sí mismo, en su acontecer fáctico, dice, indica y esboza. Este decir puro, en cuanto ejercicio de decir, de indicar, es un acontecimiento performativo irreducible a la comunicación escrita.

Según Heidegger, en una referencia velada a planteamientos centrales del Fedro, Sócrates es el pensador más puro de Occidente precisamente porque no escribió nada ${ }^{5}$. Así, la escritura se presenta en contraposición a la viva voz, a la voz encarnada al hablar, como una especie de refugio que protege de la corriente del pensamiento:

$[\mathrm{P}]$ ues quien empieza a escribir desde el pensamiento ha de parecerse inevitablemente a los hombres que se esconden en el sotovento [Windschatten], para ponerse a cobijo de un viento demasiado fuerte. Y sigue siendo el secreto de una historia todavía oculta el hecho de que todos los pensadores de Occidente después de Sócrates, sin menoscabo de su grandeza, tuvieron que ser fugitivos (Flüchtlinge) de ese tipo (Heidegger, 2002, p. 20; 2005a, p. 80).

En este orden de ideas, lo que define a Sócrates como el pensador más puro de la historia de Occidente deriva de su capacidad para exponerse a lo que urge pensar en la corriente misma de la vida, sin la distancia y sin el soporte que proporciona la escritura, a diferencia de lo que hacen sus fugitivos sucesores: los fugitivos que aparecen bajo esta luz como refugiados, desplazados, exiliados fuera de las corrientes que mueven in situ al pensamiento. En síntesis, la singularidad del pensar socrático se hace patente en su gestualidad, en el hecho de que es performativo y situado, en su fidelidad al devenir sensible del habla. Cabe aclarar que, en tanto se enfatiza lo gestual del habla y, de este modo, su carácter abierto, extático, la reivindicación de la voz no implica necesariamente la reincidencia en

5 Una referencia explícita al Fedro de Platón, en consonancia con los argumentos esbozados en estas lecciones en contra de la escritura alfabética se encuentra en las lecciones sobre las Cartas sobre la educación estética de Schiller. Véase Heidegger, 2005b, pp. 83-86. 
una metafísica de la presencia, aunque sí una reivindicación de la vida como motivo del pensamiento ${ }^{6}$.

De hecho, en el contexto de estas mismas lecciones, Heidegger proporciona una indicación vicaria según la cual lo que mueve al pensamiento, es decir, la corriente que lo arrastra, es la vida ${ }^{7}$. En referencia al poema de Hölderlin, "Sócrates y Alcibíades", se cita el verso que reza: "[Q]uién lo más profundo ha pensado, ama lo más vivo" (Wer das Tiefste gedacht, liebt das Lebendigste) (Heidegger, 2002 , p. 22; 2005b, p. 25). Lo que se define en este caso no es la pureza del pensamiento sino su profundidad, que está en correlación con la expresión superlativa de la vida: lo más vivo es aquello que arrastra con más fuerza, esto es, hacia lo más profundo, al pensamiento. Sócrates es retratado, así, como el pensador más puro en tanto que más profundo, es decir, como el que ama, busca, desea la modalidad más intensa de la vida, su máxima expresión: su virtud o excelencia, podríamos añadir aquí, buscando un acercamiento más concreto al lenguaje de los diálogos platónicos.

En este punto cabe añadir que Heidegger, años más tarde, en la década de los sesenta, en una sesión de los seminarios de Zollikon (1987; 2013), al referir a una anécdota narrada por Jenofonte, sugiere que Sócrates es el más grande pensador de Occidente no solamente porque no escribió nada, sino porque buscó decir siempre lo mismo acerca de lo mismo, que, según la lectura de Heidegger, es lo más difícil: el decir tautológico. Este es el decir que no dice nada en tanto que trata de acceder a las cosas mismas, de abrirse camino metódicamente a los fenómenos y a los presupuestos desde los cuales estos son demostrables, fiables, creíbles.

6 Precisamente, la lectura de Derrida (1987, p. 175) sobre estos pasajes sugiere que al enfatizar la auto-afección de la mano en el gesto, Heidegger permanece preso en una metafísica de la presencia.

7 Cabe referir aquí al análisis del tema de la vida en Heidegger realizado por David Farrell Krell (1992), que muestra que a pesar de sus esfuerzos por evitar toda referencia concreta a la vida, particularmente después de Ser y tiempo, el pensamiento de Heidegger se encuentra con frecuencia acosado por el fantasma de esta noción, siempre elusiva frente a cualquier intento de categorización metafísica.

8 Como bien lo señala Patrick Baur (2012, p. 86) en su análisis del “decir tautológico" de Heidegger ["Tautismus"], este define una característica fundamental de su fenomenología que remite, a su vez, a la "dificultad" como condición de acceso a las cosas mismas: solamente bajo el clima afectivo de lo "difícil" es posible mantenerse en el camino de un retorno a las cosas mismas. 
Esta referencia armoniza, parcialmente, con lo que se dice en el Gorgias, en el pasaje en que Calicles acusa a Sócrates de decir siempre lo mismo porque sus discursos son repetitivos, siempre vuelven sobre los mismos ejemplos ordinarios. En este caso, Sócrates insiste en que no solamente dice lo mismo, sino acerca de las mismas cosas, a diferencia de Calicles que siempre dice cosas distintas (Gorgias 490e-491a). Así, Sócrates resalta irónicamente cuán importante es concentrar el pensamiento en el examen consistente de unos pocos temas que se caracterizan por ser inagotables, y que refieren a la constelación de problemas relacionados con la virtud, el saber, el lenguaje, la enseñanza, la técnica y, eminentemente, la justicia y el saber político. La ironía socrática muestra, indirectamente, cuán difícil es clarificar nuestro pensamiento sobre la justicia y el bien político, pero la interpretación heideggeriana no presta atención a estos temas, al menos no explícitamente.

En efecto, según hemos visto, al caracterizar a Sócrates, Heidegger utiliza expresiones superlativas, habla de lo más puro, lo más profundo, lo más vivo, lo más difícil, y con ello parece retomar la orientación del pensamiento socrático, que supone la discriminación del valor de distintos modos de ser y la búsqueda constante de lo que significa la virtud y la excelencia de la vida humana. Sin embargo, en esta caracterización no se examina específicamente lo que significa la orientación moral y política, del pensamiento de Sócrates desde el punto de vista de la temática del gesto.

No es nuestra intención profundizar en una discusión detallada de las razones por las cuales, en este caso en particular, Heidegger evita explorar el problema de lo político; basta con señalar algunos puntos que se vislumbran como problemáticos, y que requieren de un examen detenido de acuerdo al horizonte interpretativo que se abre con el análisis de Heidegger. Los puntos en cuestión son los siguientes: que Sócrates indaga sobre la vida y el ser de las cosas en el horizonte de lo que significa la virtud política, y que en el curso de la reflexión heideggeriana sobre el decir, el gesto, el habla, si bien hay puntos que, como veremos, tocan aspectos centrales de la figura de Sócrates, se omite toda referencia al hecho de que el pensamiento socrático se caracteriza por una particular preocupación por la virtud política y, más aún, por el lenguaje político.

A la luz de estas indicaciones preliminares, en lo que sigue exploraremos lo que significa el carácter político del pensamiento de Sócrates, considerando lo 
que ha sido omitido en la reflexión heideggeriana, teniendo en cuenta los planteamientos ya esbozados sobre el decir gestual y el silencio.

2. Sócrates, el habla y la virtud

Un RASGO DISTINTIVO DE SócrATES ERA, ciertamente, su renuencia a escribir' ${ }^{9}$, pero no precisamente el callar -como lo sugiere Heidegger-, pues veía en el hablar mismo una virtud o, más aún, el camino fundamental para la virtud, para la excelencia humana. De esta manera, la virtud se presenta como la fuente por excelencia del pensamiento, el tema que da, inagotablemente, de qué hablar. Parece, entonces, que si bien el decir socrático no dice nada en tanto que tiene un carácter tautológico, aspecto enfatizado en la interpretación heideggeriana ya examinada, al volver incesantemente sobre lo mismo revela nuestro vínculo ineludible con el lenguaje, esto es, el hecho de que nuestros comportamientos, o gestos, como diría Heidegger, están permeados por el lenguaje de manera integral. En otros términos, no podemos evitar que lo que hacemos diga algo, signifique algo, y, asimismo, no podemos evitar que lo que decimos, al ser dicho, tenga el impacto de una acción. Sin embargo, no toda acción reclama de la misma manera su consumación en la palabra hablada, pública, explícita, dialógica; las acciones virtuosas, la acción justa, por ejemplo, son tales en virtud del logos, la razón y el discurso que las acompañan públicamente: un ser humano no puede juzgar o ser juzgado, en la pretensión de hacer justicia, sin que el juicio se haga explícito en la palabra, en la argumentación. Así pues, al volver repetidamente sobre temas como la virtud, la justicia y el bien, no se trata tanto de llegar a una definición teórica-abstracta, o de pretender retóricamente transformar al interlocutor en su interior, sino de darle vida a la virtud, de invocar su aparición, pues sin la palabra, el logos, el pensamiento, el ser humano no alcanza en sus acciones la excelencia, la virtud, la perfección. Para evaluar en detalle este planteamiento

9 En el Fedón (60c-61b) se encuentra lo que parece una excepción a esta caracterización, pues allí se narra que mientras espera en su celda el momento de la ejecución, Sócrates dedica su tiempo a componer poemas "versificando las fábulas de Esopo". Cabe aclarar que en este pasaje se describe

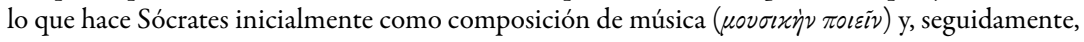

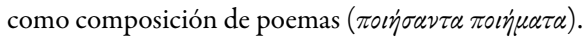


me centraré en un análisis del siguiente pasaje de la Apología, en el que se hace referencia específicamente a la relación entre palabra y virtud:

Quizás diga alguno: «¿Pero no serás capaz de vivir alejado de nosotros en silencio y llevando una vida tranquila?» Persuadir de esto a algunos de vosotros es lo más difícil. En efecto, si digo que eso es desobedecer al dios y que, por ello, es imposible llevar una vida tranquila, no me creeréis pensando que hablo irónicamente. Si, por otra parte, digo que el mayor bien para un hombre es precisamente este, tener conversaciones [hacer discursos] cada día acerca de la virtud y de los otros temas de los que vosotros me habéis oído dialogar cuando me examinaba a mí mismo y a otros, y si digo que una vida sin examen no tiene objeto vivirla para el hombre, me creeréis aún menos. Sin embargo, la verdad es así, como yo digo, atenientes, pero no es fácil convenceros $^{10}$ (Apología 37e-38a).

En este pasaje, que sigue a la argumentación sobre cuál pena sería justa en su juicio, Sócrates afirma en primera instancia que lo más difícil $(\chi \alpha \lambda \varepsilon \pi \omega \dot{\tau} \tau \alpha \tau o \nu)$ es persuadir a algunos de sus interlocutores de que no le es posible llevar una vida tranquila y en silencio, por ejemplo, en el exilio ${ }^{11}$. La dificultad deriva de que la labor de Sócrates en la polis, que consiste en interpelar a sus conciudadanos y no abandonarlos a su suerte en lo relativo a cuestiones sobre la virtud, el saber y la vida buena, obedece a un comando divino.

No es, pues, una condición meramente humana, convencional, la que le impide a Sócrates permanecer en silencio. En efecto, ante una simple convención parece sencilla la desobediencia y, asimismo, la ironía: es algo que podría no

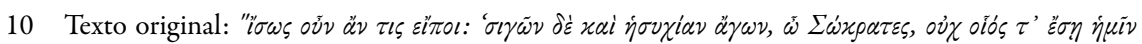

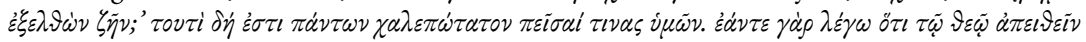

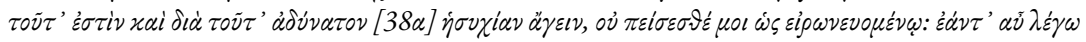

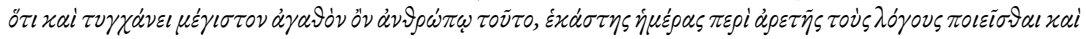

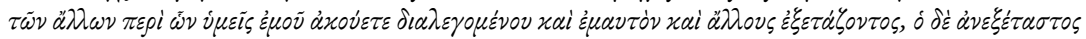

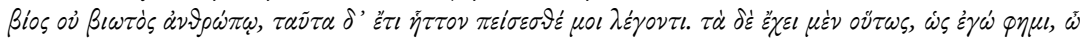

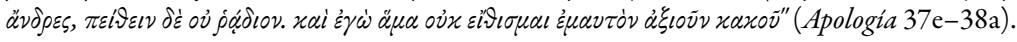

11 En relación con este planteamiento, cabe resaltar la oposición entre lo que Sócrates considera aquí como lo más difícil, a saber, persuadir a los otros de la verdad, y lo que Heidegger subraya como lo más difícil, esto es, decir lo mismo acerca de lo mismo. Esta oposición es importante porque en últimas la dificultad que subraya Sócrates en este pasaje refiere a una dificultad que se da en el uso público de la palabra y que se considera, probablemente, inherente al mismo. Así pues, lo más difícil no es callar para decir lo mismo, es hablar a pesar de no ser comprendido, y persistir en este esfuerzo. 
tomarse en serio. Sócrates, sin embargo, subraya que no está siendo irónico, y concluye el pasaje afirmando que dice la verdad.

El pensador responde, pues, a una exigencia de la mayor seriedad, ineludible, que lo conmina a no sustraerse de la vida pública, a hablar con la verdad, con franqueza, a no caer en la tentación de callar para, simplemente, mantenerse con vida. Por otra parte, en consonancia con el mandato divino, Sócrates afirma que el bien mayor, superlativo ( $\mu \dot{\varepsilon} \gamma l \sigma \tau o v \dot{\alpha} \gamma \alpha \dot{\gamma} \dot{\nu}$ ) para el ser humano, consiste en hacer discursos sobre la virtud y los otros temas sobre los que se le ha escuchado hablar de manera habitual: la referencia es, como ya lo hemos sugerido, a temas como el bien, el saber, la justicia y la vida buena, entre otros también vinculados al problema de la virtud. En este planteamiento, sin embargo, hay un aspecto que llama especialmente la atención y que no debemos pasar por alto: Sócrates no dice que el mayor bien para el hombre es ser virtuoso, sino que el mayor bien reside, literalmente, en

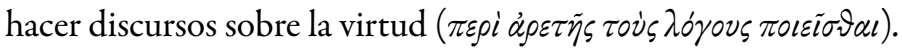

Así pues, en el pasaje citado se pueden distinguir dos momentos fundamentales que, por su complejidad, ameritan una extensa explicación. En primer lugar, al hablar de lo que dice el dios, que se presenta como un imperativo y en cierto sentido como una fatalidad, Sócrates sugiere que su labor ha de ser concebida como una forma de piedad, de manera que no puede renunciar a la palabra, callar la verdad y no hacer aquello de lo cual está íntimamente convencido, sin al mismo tiempo justificar una de las acusaciones de la que es víctima, a saber, la de impiedad. En relación con este punto, cabe suponer, además, que, en tanto Sócrates ha justificado su labor como un bien efectivo a la polis, que merecería incluso el gran honor de la manutención en el Pritaneo, se trata de una labor que responde a una exigencia eminentemente política ${ }^{12}$. De acuerdo con esto, en la posición socrática no solamente resuena el ideal ateniense de la parresía, el hablar franco, que en el Gorgias se concibe como condición de posibilidad del arte político -y que para

12 A la pregunta sobre cuál pena considera que sería justa, Sócrates responde, no sin ironía, que en vez de una pena merece una recompensa, pues por sus servicios a Atenas se le debería mantener en el Pritaneo, precisamente el mayor honor que la ciudad podría otorgar a un ciudadano. 
los atenienses era una virtud política ${ }^{13}$, si bien no siempre contemplada- ${ }^{14}$, sino que, en un sentido más profundo, se sugiere que una responsabilidad fundamental con la polis, una responsabilidad política, en últimas, se presenta como una exigencia sagrada, inviolable, es decir, se impone con un poder inconmensurable con respecto al poder humano. Así, la responsabilidad para con los otros es irreductible a lo instrumental -no se puede acomodar a fines particulares-y no se circunscribe al dominio de la voluntad individual. En otros términos, poniendo entre paréntesis la referencia a lo divino, la posición socrática muestra que los valores e imperativos políticos fundamentales tienen una vigencia y legitimidad que, si son asumidas con el debido rigor, suponen una autoridad inapelable.

Sócrates afirma que le es imposible no seguir el comando divino. Teniendo en cuenta que seguidamente él cualifica su labor, aquella que realiza obedeciendo al dios, como el mayor bien para el ser humano, es decir, como un bien universal -un bien común, político-, no un bien particular, entonces parece que el comando divino es imperativo para cualquiera. El término usado en este caso es ả $\delta \dot{v} v \alpha \tau o v, \mathrm{y}$ alude a una imposibilidad en el sentido de incapacidad, impotencia o carencia de fuerza. De este modo, al establecerse un vínculo entre lo sagrado y lo político, se sugiere que en el ámbito de lo político lo indebido se amalgama de alguna manera con lo imposible: lo que no se debe hacer es algo que, en últimas, no somos capaces de hacer o, mejor aún, no está en nuestro poder cambiar. Lo indebido no es, jamás, algo que uno simplemente decide ${ }^{15}$. En síntesis, la posición de Sócrates en

13 Según lo muestra el análisis de Cristina H. Tarnopolsky (2010, pp. 201-205), la parresía era un ideal democrático ateniense en consonancia con la concepción platónica de la política. Este ideal no solamente era una virtud que se esperaba del orador, y que supone el valor para decir la verdad de las propias convicciones, sino que además suponía una cierta virtud del auditor, en su capacidad para aceptar las críticas y revisar las propias opiniones. Como es evidente en diálogos platónicos como el Gorgias, o la misma Apología, el pueblo ateniense no siempre era consecuente con lo que implicaba esta virtud.

14 Efectivamente, en el Gorgias Sócrates sugiere que las tres condiciones para examinar el alma son "ciencia, buena voluntad y franqueza" (487e). Puesto que el examen del alma se concibe en este diálogo como función del arte político, es lícito suponer que la franqueza es condición para el ejercicio del tipo de política que defiende Sócrates. Igualmente, cabe resaltar que, según se muestra en el mismo diálogo, estas condiciones rigen también en una verdadera amistad. El arte político es, entonces, un arte que supone relaciones de amistad.

15 En otros términos, según lo explicaremos más adelante, nuestra interpretación supone que aquello que se valora como correcto o incorrecto, bueno o malo, en el seno de una comunidad no es algo que puedan modificar los individuos caprichosamente. 
relación con el carácter sagrado de su responsabilidad con la polis se puede replantear de la siguiente manera: no es posible, en realidad, callar la verdad, el ser humano no puede evitar hablar de lo que le concierne, es imposible e indebido, en la polis, en tanto que se es ciudadano, permanecer en silencio ${ }^{16}$. En otras palabras, si la polis es el espacio de lo común, entonces ella exige comunicabilidad ${ }^{17}$.

Sócrates subraya que la verdad que él dice es difícil de aceptar, o difícil de creer, para algunos de sus interlocutores. Esta indicación revela una situación paradójica, pues, por una parte, lo que Sócrates afirma en este caso corresponde a un saber verdadero que, además, es un saber que refiere a la verdad, a la necesidad de decir la verdad, de manifestarse, de hablar y de no callar. En otros términos, Sócrates afirma que es verdad que se debe decir la verdad, y esta no es una mera convención u opinión sino un principio ineludible. Sin embargo, por otra parte, lo que dice Sócrates es una verdad que no persuade fácilmente, esto es, se trata de una verdad que no es evidente o conceptualizable, que no coincide necesariamente con lo que se percibe en los hechos, y que parece posible, incluso fácil, ocultar y reprimir. En efecto, lo que asumen los interlocutores de Sócrates, aquellos no persuadidos por su discurso, es que la verdad se puede decir o no según convenga porque de facto podemos decirla o no según convenga, mientras que Sócrates intenta mostrar que aquello que en principio es inviolable, sagrado, universal, no puede, en realidad, subordinarse a un ejercicio particular de la voluntad. En otras palabras, Sócrates se atiene al pie de la letra a lo que implica la legitimidad de un principio moral y político de carácter universal: que mintamos a voluntad no cambia en nada el hecho de que, en nuestras relaciones sociales y políticas -e incluso en nuestros comportamientos en general-, presuponemos, por ejemplo, como deseable la verdad, e indeseable la mentira, en consonancia

16 De esta manera, la intervención de Sócrates enfatiza que la verdad es aquello que no puede no ser visto, o dicho, es aquello que ya se hace patente en lo que dicen nuestras actitudes o comportamientos, independientemente de lo que queramos o no decir explícitamente, a voluntad.

17 En este punto, aplicando algunos planteamientos de Antonio Da Re (2016) al caso de Sócrates, se podría decir que Sócrates asume lo universal como lo común de la siguiente manera: "[E]l universal como común (y no tanto como absoluto contrapuesto a relativo) es lo que permite reconocer y valorar las diferencias: si no hubiese algo común no podría haber intercambio, encuentro, comunicación, entre los sujetos, entre las culturas, entre tradiciones y concepciones diferentes de la vida" (p. 179). En este orden de ideas, se podría decir que renunciar a la comunicación, suponiendo el ideal de la parresía, es atentar contra los cimientos de la polis misma. 
con un bien común que no puede subordinarse a nuestra perspectiva particular o intereses de grupo $^{18}$.

Puesto que la posición socrática es, como se acaba de observar, difícil de creer, es útil insistir en una explicación más elaborada sobre el tipo de poder con el que se impone la verdad, tema que nos permitirá retomar la reflexión inicial sobre lo gestual. Al respecto, cabe señalar que los diálogos platónicos muestran con frecuencia que lo realmente pensado, lo que en el fondo se acepta como verdadero, suele revelarse en gestos irreprimibles; se manifiesta subrepticia pero contundentemente, en comportamientos y también en lo que ya está presupuesto en el lenguaje mismo. Los siguientes ejemplos ilustran este punto: en el Fedro, al pronunciar el primer discurso, que no dice la verdad sobre Eros, Sócrates se ve obligado a agachar la cabeza y ocultar su rostro en un gesto de autoreprobación (Fedro 237a); en el Lisis, Sócrates descubre en el sonrojarse de Hipotales el amor, no declarado abiertamente, aunque genuino, de este por Lisis (Lisis 204b); en el Gorgias Sócrates hace patente la inconsistencia de la posición de Calicles haciéndolo reconocer, a través de ejemplos que evidentemente lo avergüenzan -como el de la referencia al placer que produce rascarse, o la sodomía que, Calicles, en su particular concepción de la virilidad, no podría aceptar-, que él realmente piensa que no todo placer es igualmente bueno (Gorgias 494e). En todos estos casos prevalece el reconocimiento de un saber de trasfondo, de principios que ya se han aceptado sobre lo que es bello, deseable, bueno, entre otros, pero que, en virtud de su profundo arraigo e intimidad son, con frecuencia, difíciles de percibir y de explicitar. La posición socrática implica, de esta manera, que los principios fundamentales que constituyen una comunidad política están ya siempre dados o presupuestos como un saber incorporado, un saber sedimentado -esto es, una cierta racionalidad, discursividad o comunicabilidad, y no un mero instintoque se hace patente en nuestros comportamientos, en nuestros gestos $\mathrm{y}$,

18 Este argumento replica planteamientos esbozados al final del libro sexto de la República sobre la inagotabilidad del bien que, según lo enfatiza Antonio Da Re, pueden ser leídos como una instancia crítica respecto a toda pretensión de erigir un punto de vista particular como paradigma universal: “[L] a tesis, sostenida con fuerza, de la indefinibilidad de la idea del Bien es interpretada en términos críticos, como inagotabilidad del bien e imposibilidad para el hombre, finito y limitado, de poderlo comprender y expresar en su totalidad. La trascendencia del Agathon sirve para indicar que el Bien no puede ser limitado de manera inmanente por alguna doctrina particular que pretenda ser exhaustiva" (Da Re, 2016, p. 56). 
asimismo, en la dimensión gestual del lenguaje. De este modo, nuestra comunicación explícita, predicativa, conceptualizada, presupone un lenguaje más profundo, sedimentado, gestual, que es fuente de significaciones que exceden lo que queremos o creemos decir. De este modo, vemos que el argumento de Sócrates coincidiría con el planteamiento heideggeriano según el cual nuestros gestos, o nuestros comportamientos en general, están siempre atravesados por el lenguaje.

En relación con este punto es indicativo lo que se sugiere en el Libro IV de la República, en el que Sócrates hace notar que la tan buscada justicia, aquella que él y sus interlocutores están acechando como cazadores, está más cerca de lo que se cree, imperceptiblemente presente, en los discursos mismos que indagan sobre ella: “ $[\mathrm{H}]$ ace rato, $y$ ya desde el principio ( $\dot{\xi} \xi \dot{\alpha} \rho \chi \tilde{\eta} \varsigma)$, querido amigo, creo que ha estado rondando delante de nuestros pies, pero que no la hemos percibido, sino que nos hemos comportado ridículamente, como los que a veces se desesperan buscando algo que tienen en sus manos" (República 432d). En este pasaje se plantea, por una parte, que la justicia ya está presupuesta en la indagación sobre la justicia y, por otra parte, que ella resulta imperceptible precisamente por su cercanía y familiaridad ${ }^{19}$.

Este saber de trasfondo, siempre presupuesto y aceptado, corresponde, según lo que se plantea en el Gorgias, al pensar verdadero, común, el pensar que todos - por lo menos todos aquellos que participan del horizonte de una misma comunidad política-, en el fondo piensan, y que se distingue de lo que simplemente se cree, de lo que se asume como una opinión particular ${ }^{20}$. Un ejemplo específico, planteado por Sócrates en el Gorgias, de esto que realmente se piensa pero que podría fácilmente pasar inadvertido, es el de la máxima según la cual es preferible sufrir la injusticia que cometerla (Gorgias $509 \mathrm{c}-\mathrm{d}$ ).

El comando divino que obliga a la responsabilidad política de decir la verdad opera como lo que podríamos denominar un a priori práctico, un principio que está presupuesto en todo comportamiento, un saber de principios o máximas

19 En relación con este punto, y de acuerdo con la explicación que sigue, se podría decir que el análisis filosófico de la opinión es necesario precisamente porque ordinariamente pasan desapercibidos para nosotros los principios morales presupuestos en las propias acciones.

20 Nos referimos aquí a la distinción que establece Sócrates entre hacer lo que a uno le parece y hacer realmente lo que uno quiere, que es el bien (Gorgias 469e-470b). 
que no se pueden justificar o fundamentar precisamente porque se trata de principios, es decir, de la base desde la cual emerge la justificación o fundamentación, la fuente de la que emanan los discursos mismos -como se sugiere también en el contexto teórico del pasaje citado de la República-. Por su familiaridad e intimidad, se trata de principios que se anuncian como significaciones afectivas, considerando, por ejemplo, la capa afectiva y el saber de trasfondo que recubre nuestras palabras, por ejemplo, cuando se habla de lo deseable, vergonzoso, loable, repugnante, entre otros.

Aunque este a priori práctico opera siempre como un saber sedimentado que impregna nuestros comportamientos y la significación afectiva, sedimentada, heredada, de nuestro lenguaje, aquella que se resiste a la conceptualización plena quizás, por ello precisamente, lo presente Sócrates como un saber de origen divino-, esto no significa que sea inútil la tarea de intentar racionalizarlo o conceptualizarlo, al menos en parte, según las exigencias particulares del presente que se vive. Más aún, puesto que se trata de un saber sedimentado, ancestral, en cierto sentido, oculto y olvidado, que, no obstante, determina de manera fundamental nuestras relaciones sociales, nuestra vida, es necesario intentar, siempre de nuevo, hacerlo visible, darle forma, recrearlo, tal y como pretende hacerlo Sócrates en su continuo examen de la opinión de sus conciudadanos. El comando divino que sigue Sócrates puede interpretarse también en este sentido, a saber: la verdad revelada, la verdad que se presenta como principio, exige ser dicha, declarada abiertamente, apropiada en nuestro lenguaje público, vigente; el logos común, la racionalidad que nos vincula abierta y públicamente a los otros en un momento dado.

Esto nos lleva al problema que se plantea en la segunda parte del pasaje de la Apología que estamos examinando, y que refiere al lenguaje que podemos hacer, crear, poetizar sobre la virtud, a partir del lenguaje primario de esta verdad que siempre se nos impone como una significación ya dada o constituida.

Como fue indicado anteriormente, Sócrates afirma que el mayor bien para el ser humano, esto es, el mejor género de vida, consiste en hacer o producir discursos sobre la virtud. Ciertamente, con esto no se quiere significar que es más importante hablar sobre la virtud que ser virtuoso -asumiendo, entonces, que se pueden separar ambas instancias-, posición que difícilmente podríamos atribuir 
a Sócrates o a Platón. Asumimos, por el contrario, una interpretación según la cual en el hablar sobre la virtud acontece la virtud misma ${ }^{21}$.

$\mathrm{Al}$ respecto, es importante enfatizar, en primer lugar, que Sócrates complementa esta indicación sobre el mayor bien anotando que sus propios discursos, tal y como han sido escuchados por sus interlocutores, son instancias del género de discurso, y de vida, que defiende; se trata, pues, de discursos hechos como un acto público, a la vista de todos. Aunque cabe aclarar, de acuerdo con lo que el mismo Sócrates insinúa en la Apología, que no es una actividad pública y política en el sentido convencional: no se trata de discursos políticos en los tribunales, y no se dirigen a multitudes, se concentran en el diálogo con individuos.

En segundo lugar, debemos considerar que Sócrates afirma específicamente que una vida sin examen no es vida para el ser humano, no es vida humana $(\dot{\delta} \delta \dot{\varepsilon}$

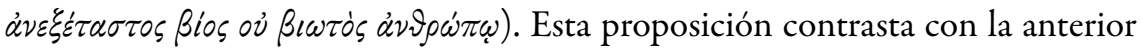
en tanto que determinación de la condición básica que define la vida humana.

Así pues, por una parte, Sócrates refiere al máximum de la vida, a la vida que conlleva el mayor bien para el ser humano y, por otra parte, refiere a la condición que define la condición básica, mínima, de la misma, aquello sin lo cual esta no puede ser asumida como humana. Llama la atención que el criterio para definir la condición básica de la vida parece extraordinario, mientras que el máximum no: una vida que se vive sin más, sin examen, no es vivible, no vale la pena, mientras que el mayor bien consiste, "simplemente", en hablar, hacer discursos, sobre la virtud. Una lectura atenta del pasaje demuestra, sin embargo, que la vida dedicada a hablar de la virtud y la vida dedicada al examen de sí y de los otros se sobreponen. El mínimum de la vida es ya su máximum, la vida que el ser humano

21 En el contexto de una discusión teórica muy distinta, esto es lo que sugiere Matt Dillon en su lectura de lo que significa la virtud socrática en el Menón al compararla con la orientación de la filosofía de Maurice Merleau-Ponty: "La ignorancia socrática no es ni un mero instrumento pedagógico ni una forma de falsa modestia ni la confesión, por parte de Sócrates, de que él no sabe nada de la

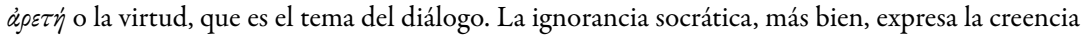
de que la incesante búsqueda de la verdad que la figura de Sócrates ejemplifica es, en sí misma, la excelencia humana o la virtud sobre la que se discute"([S]ocratic ignorance is neither a mere pedagogical device nor a form of false modesty nor an admision by Socrates that he really knows nothing of apstท or virtue, the theme of the dialogue. Socratic ignorance is, rather, an expression of the belief that the ceaseless striving for understanding portrayed in the figure of Socrates instantiates, itself, the human excellence or virtue under discussion) (Dillon, 1988, p. 3). 
puede vivir, soportar en sus límites, exige siempre el mayor bien posible, está impelida a la búsqueda de este paradigma de perfección.

Retomemos el planteamiento central del pasaje. Sócrates comienza afirmando que el mayor bien es hablar sobre la virtud, examinándose a sí mismo y a los otros, para añadir seguidamente que sin este examen la vida no es vivible. En otras palabras, el hablar sobre la virtud se presenta como encarnación de la virtud, pero se trata, por supuesto, de una encarnación siempre in fieri, en proceso, pues el hablar sobre la virtud no es un decir cualquiera, es un decir que indaga, examina ${ }^{22}$. El discurso sobre la virtud se correlaciona así, intrínsecamente, con un comportamiento específico: la indagación o el examen. Esta conclusión contiene una paradoja. La vida virtuosa solamente es tal si se asume como una disposición constante e incesante a la búsqueda de la virtud y la excelencia; por lo tanto, no se puede asumir a sí misma como efectivamente virtuosa, como instanciación de la perfección. En este orden de ideas, en tanto el comportamiento que constituye la virtud es un comportamiento que no debe alcanzar su objetivo, no puede ser satisfecho, el mayor bien para el ser humano conlleva su pérdida, implica el reconocimiento anticipado de un fracaso: lo buscado, la vida buena, se escapa siempre, permanece como una latencia, en potencia. Por ello, vivir al máximo supone de alguna manera menospreciar toda configuración actual, toda instanciación concreta, fáctica, de la vida.

En síntesis, lo máximo que puede hacer un ser humano es dedicar su vida a indagar, buscar, examinar, no a encontrar, tener y ser. Más precisamente, lo máximo que puede hacer es buscar o examinar en el lenguaje, en el decir, en el habla, una significación que siempre se difiere, que nunca se da en el dominio vital de los hechos, de lo que no aparece como puro lenguaje. De este modo, por ende, el hablar sobre la virtud, que además se presenta en sí mismo como instanciación de la virtud, se revela también como un hablar, un modo de ser o existir, de comportarse y actuar, que permanece rigurosamente en el medium del habla, que no pretende hacer nada más allá del decir, esto es, que solamente indica, señala, una significación que, perennemente, se sustrae, que resulta inagotable.

22 Asumiendo, pues, que mientras estamos vivos nunca llegamos al estado de sabiduría plena, la virtud humana, la excelencia humana, en tanto que humana y no divina, se restringe a los límites del examen, de la búsqueda, no del hallazgo. 


\section{La virtud, el poder político y el medium del lenguaje}

SóCrATES SUGIERE, PUES, QUE la excelencia humana, la justicia, la virtud, residen en el medium del puro lenguaje, en lo que el lenguaje dice desde sí mismo. La virtud, la perfección accesible al ser humano, es una perfección que solamente se nos da cuando actuamos con palabras. Así, lo máximo que puede hacer un ser humano se restringe a lo que hacen las palabras desde su precaria materialidad. De esta manera, en el pasaje que estamos analizando de la Apología se esboza una compleja relación entre palabra y acción, una relación que no puede ser pensada como oposición: el ser humano alcanza la virtud, llega a su máxima expresión, al limitar su vida al minimum de acción que se da en el habla. En relación con esta lectura, cabe recordar que Sócrates recalca en la República, al final del Libro II, que la justicia puede hacerse visible si se observa una polis en vez de un individuo y, particularmente, si se observa una polis que se constituya como tal en el lenguaje, en la palabra (República 369a).

Para concluir profundicemos en este planteamiento. Según lo sugiere Sócrates en sus constantes referencias al zapatero, al artesano e incluso al médico, aquellas que motivan la queja de Calicles sobre lo repetitivo de los discursos de Sócrates, cuando se trata de la justicia, el bien, la virtud, jamás observamos resultados visibles, concluyentes o verificables. De hecho, la analogía que se establece en el Gorgias entre el político y el médico alude a que aquel saber se orienta a la dimensión invisible, intangible del ser humano, esto es, aquella que, en principio, no se puede instrumentalizar u objetivar: el dominio elusivo del alma. De acuerdo con esto, se podría pensar que el estado del alma en relación con la virtud, la justicia o el bien se revela de alguna manera, así sea vicaria y elusivamente, en el lenguaje, en la expresión de las creencias y opiniones, incluyendo, como hemos visto, la modalidad del lenguaje que es gesto. Esta idea supone que el lenguaje es vehículo de una significación extrínseca. Sin embargo, como bien se muestra en los diálogos platónicos, la salud del alma coincide con la consistencia del discurso: consiste en reconocer lo que dicen las palabras mismas, los vínculos que establecen entre sí palabras tales como "bien", "justicia" y "virtud". El saber del verdadero político es, contrariamente a lo que sucede en cualquier saber técnico ordinario, un saber paradójico cuyo poder no se evalúa a partir de la realización de fines, sino por la rigurosa atención a los medios en cuanto tales. En otros 
términos, el político como médico del alma debe restringir su examen a lo que se hace manifiesto en el logos, en la medialidad de una expresión, en el despliegue del discurso. Por tanto, el médico del alma, a diferencia del médico del cuerpo, no puede, y no debe, pretender ninguna operatividad efectiva en lo que sería el ser de la persona o, incluso, de la polis, más allá de lo que el lenguaje puede hacer por sí mismo y en sí mismo.

En el Gorgias se desarrolla, indirectamente, esta intuición. Según se muestra en el curso del diálogo, Sócrates trata por todos los medios que proporciona el lenguaje mismo -el mito, la exhortación directa, la refutación lógica- de convencer a Calicles de que es mejor ser justo que injusto. Calicles se ve obligado a reconocer esto, pero solamente en el lenguaje, en la performance, en virtud de la consistencia lógica del diálogo: dice por decir aquello que exige el discurso mismo, y cuya verdad él no logra, a pesar de sus esfuerzos, alterar. Además, en el curso de una discusión sobre el poder de la retórica en contraposición al poder de la verdadera justicia, Sócrates muestra cuán paradójica, e imposible, es la situación de quien intenta yuxtaponer la efectividad de hecho y el ideal de la justicia, particularmente en relación con el problema del castigo.

Cuando en el Gorgias se alude al castigo, se lo muestra como un bien que, en últimas, el castigado debe asumir como tal (Gorgias 525a-b). Esto significa, aunque Sócrates no lo haga explícito, que el castigo es siempre innecesario como vía de hecho o, por lo menos, está subordinado a un ejercicio de pura comunicación: si el condenado a un castigo lo asume como un bien, como un castigo justamente merecido por un mal o un daño cometido, entonces el condenado se revela, por lo menos en el lenguaje o la expresión, como justo, como bueno, como virtuoso, lo que, de hecho, anularía el sentido y necesidad del castigo; más aún, lo haría injusto. $\mathrm{Si}$, por el contrario, el acusado no admite sinceramente su culpa, el castigo se revela inútil, sin sentido. En este caso se hace patente, irónicamente, que es imposible verificar el estado del alma en relación con la virtud más allá de lo que el lenguaje mismo revela respecto del estado en cuestión: el castigo solamente tiene sentido en la medida en que el acusado responde en sus gestos, palabras o expresiones al sentido o significado del castigo, esto es, a lo que el castigo pretende decir o enseñar. Sin embargo, nunca es posible constatar exhaustivamente la veracidad de esta expresión, así como tampoco es posible definir exhaustivamente el uso de una palabra o los límites del lenguaje. 
En este orden de ideas, la cura que ofrece el político entendido como médico del alma es, pues, una cura que solamente se da en el medium del habla, es una cura que se presenta en últimas como cuidado del lenguaje, de la expresión como expresión. Se cuida el alma, entonces, en la medida en que se cuida o preserva lo que se dice, asumiendo la fatalidad de que esta tarea nunca alcanza un fin o una consumación última.

En conclusión. Sócrates se resiste a callar, y dice la verdad que es inevitable, irreprimible; sin embargo, a la luz de lo que acabamos de plantear, lo que significa en este caso decir la verdad es, en últimas, ser fiel a la verdad del decir, a su acontecer como puro acto de expresión, como un gesto cuya finalidad o referencia última se sustrae indefinidamente. En efecto, no es posible hablar de la virtud, o de la justicia, o del bien, sin constatar al mismo tiempo su ausencia, su lejanía, su elusiva presencia en la vida y en los hechos. Así, las palabras que no son más que palabras, acciones precarias y sutiles que se lleva el viento, hacen palidecer el valor de los hechos concretos y, más aún, de la vida: los hechos y la vida nunca corresponden a la plenitud que se vislumbra en lo que hacen las palabras, si bien estos mismos hechos, y esta vida, solamente brillan, se hacen patentes como algo valioso -o incluso como "algo" en general-, al ser capturados en las redes del lenguaje o, más precisamente, del lenguaje sobre la virtud. Así, una vida que solamente es buena, vivible, cuando se dedica al habla, al discurso, a la palabra que es examen, es una vida que está en falta, en deuda, en retraso, respecto de su máxima expresión, es la vida condenada al examen porque nunca es lo que debería ser. De este modo, Sócrates muestra que nunca estamos a la altura de la palabra, si bien la palabra tampoco puede, jamás, satisfacernos. La virtud consiste, pues, en vivir conforme a la precariedad de la palabra.

Esta parece una conclusión que no hace justicia a la virtud y la justicia, pues si nos vemos abocados a investigar sobre la virtud y la justicia es en razón de nuestro deseo de llegar a ser efectivamente justos y virtuosos, o hacer de nuestra comunidad una comunidad realmente justa. Sin embargo, y lo que sigue debe asumirse con el tono de una pregunta, cabe considerar si dados nuestros límites y nuestra naturaleza, cuando se trata de los ideales del bien, la virtud y la justicia es preferible hablar que hacer o, más bien, limitar el hacer al hablar, considerando que esto no sería poco si en general fuésemos fieles a lo que dicen las palabras, manteniéndonos en su dominio, y asumiendo que el acontecer efectivo de la 
justicia y el bien político, lo que sería la justicia y el bien en sí, sería algo que, en principio, solamente podría decretar un dios, esto es, quien tuviera el poder de hacer que el mundo sea según su voluntad. Quizás el problema del mal, la injusticia, o incluso la impiedad, surja precisamente del desconocimiento de lo que significa este morar en el lenguaje, y de la confusión entre lo que está en el dominio del lenguaje y lo que no, es decir, lo que está en el dominio de la vida, y lo que no. Al respecto, según hemos visto, la conclusión, inequívoca, de Sócrates es que el dominio del verdadero político, su poder, se restringe a lo que se puede indagar en el lenguaje y lo que se puede hacer en la palabra, es decir, hacer discursos, proferir palabras.

\section{Referencias}

Baur, P. (2013). Phänomenologie der Gebärden. Freiburg/München: Verlag Karl Alber.

Da Re, A. (2016). Las palabras de la ética. (Trad. G. Gómez Pérez). Avarigani: Madrid.

Dillon, M.C. (1988). Merleau-Ponty's Ontology. Bloomington: Indiana University Press.

Derrida, J. (1987). Geschlecht II: Heidegger's Hand. En John Sallis (Ed.), Deconstruction and Philosophy (161-196). Chicago: The University of Chicago Press.

Heidegger, M.. (2005a). ¿Quésignifica pensar? (Trad. R. Gabás). Madrid: Trotta.

Heidegger, M. (2013). Seminarios de Zollikon. (Trad. A. Xolocotzi Yáñez). México D.F.: Herder.

Heidegger, M. (2005b). Übungen für Anfänger. Schillers Briefe über die äesthetische Erziehung des Menschen. Wintersemester 1936/37. Marbach am Neckar: Marbacher Bibliothek. Deutsche Schillergesellschaft.

Heidegger, M. (2002). Was heisst Denken. Gesamtausgabe Bd.8. Frankfurt am Main: Vittorio Klostermann.

Heidegger, M. (1987). Zollikoner Seminare. Frankfurt am Main: Vittorio Klostermann. 
Krell, D. F. (1992). Daimon Life: Heidegger and Life-Philosophy. Bloomington: Indiana University Press.

Plato. (1903). Apology. Platonis Opera. Oxford University Press. Disponible en línea: http://www.perseus.tufts.edu/hopper/text?doc=Perseus\%3Atext\%3 A1999.01.0169\%3Atext\%3DApol.

Platón. (1985). Apología. (Trads. J. Calonge Ruiz, E. Lledó Iñigo, C. García Gual). En Diálogos I.

Platón. (1988). Fedón. (Trad. C. García Gual). En Diálogos III. Madrid: Gredos.

Platón. (1967). Gorgias. (Trad. A. Cappelletti). Buenos Aires: Editorial Universitaria de Buenos Aires (EUDEBA).

Platón. (1988). República. (Trad. C. Eggers Lan). En Diálogos IV. Madrid: Gredos.

Tarnopolsky, C.H. (2010). Prudes, Perverts, and Tyrants. Plato's Gorgias and the Politics of Shame. Princeton: Princeton University Press. 